\title{
Quantum Fluctuations and Noise in Parametric Processes. I.
}

\author{
W. H. Loutsell and A. Yariv \\ Bell Telephone Laboratories, Murray Hill, New Jersey \\ AND \\ A. E. Siegman \\ Stanford University, Stanford, California \\ (Received June 27, 1961; revised manuscript received August 31, 1961)
}

\begin{abstract}
A quantum mechanical model for parametric interactions is used to evaluate the effect of the measuring (amplifying) process on the statistical properties of radiation. Parametric amplification is shown to be ideal in the sense that it allows a simultaneous determination of the phase and number of quanta of an electromagnetic wave with an accuracy which is limited only by the uncertainty principle. Frequency conversion via parametric processes is shown to be free of zero-point fluctuations.
\end{abstract}

\section{INTRODUCTION}

$\mathbf{P}$ ARAMETRIC interactions, which were first studied by Faraday and Lord Rayleigh in the nineteenth century, are now receiving renewed attention which is probably due to their successful utilization as microwave amplifiers.

The classical characterization of parametric processes is that of singly or multiresonant systems in which an energy storage parameter is harmonically modulated. This modulation can cause a periodic energy exchange between the resonant systems or a continuous transfer of energy from the modulation source to the resonant systems.

When the resonant systems are electromagnetic modes the second of the two processes described above can be used for coherent amplification of radiation, i.e., amplification in which both the phase and the amplitude of the incoming electromagnetic wave are reproduced. Any amplification process, of necessity, introduces a certain amount of uncertainty into the determination of the phase and amplitude thus degrading the amount of information carried by the wave. In the case of maser amplifiers, which have been investigated extensively, ${ }^{1-7}$ it was shown that the uncertainty $\Delta n$ in the number of photons in the input wave and the uncertainty $\Delta \varphi$ in its phase as deduced from an examination of the amplified output satisfy

$$
\Delta n \Delta \varphi=\frac{1}{2},
$$

which is the minimum amount allowed by the uncertainty principle, provided the average number of quanta is large compared with 1 . This fact qualifies the maser amplifier as an "ideal" phase sensitive amplifier.

We are able to show that the lossless parametric

\footnotetext{
${ }^{1}$ K. Shimoda, H. Takahasi, and C. H. Townes, J. Phys. Soc. Japan 12, 686 (1957).

2 R. Serber and C. H. Townes, Quantum Electronics-A Symposium, edited by C. H. Townes (Columbia University Press, New York, 1960), p. 233.

${ }^{3}$ R. V. Pound, Ann. Phys. 1, 24 (1957).

${ }^{4}$ M. W. P. Strandberg, Phys. Rev. 106, 617 (1957).

${ }^{5}$ M. W. Muller, Phys. Rev. 106, 8 (1957).

${ }^{6} \mathrm{~J}$. P. Gordon, Quantum Electronics Symposium, Berkeley, California, 1961 (unpublished).

${ }^{7}$ A, E. Siegman, Proc. Inst. Radio Engrs. 49, 633 (1961).
}

amplifier is also an "ideal" phase sensitive amplifier. This is done by the use of a quantum mechanical model for parametric processes which in the classical limit yields all the known classical features of parametric amplifiers. This quantum mechanical model is in fact so simple, especially when compared to that of the negative-temperature (maser) type of amplification, that it makes the parametric amplifier an attractive model for the study of the statistical properties of phase-coherent amplification and the limiting uncertainties imposed on amplitude and phase measurements by quantum mechanical fluctuations.

The formalism of field quantization is used to obtain a solution for the time-dependent annihilation operators $a_{1}(t)$ and $a_{2}(t)$ of the resonant modes at $\omega_{1}$ and $\omega_{2}$, respectively. This is also done for the creation operators $a_{1}^{\dagger}(t)$ and $a_{2}^{\dagger}(t)$. The operators are then used to calculate the expectation values for the number of photons $\left\langle a^{\dagger}(t) a(t)\right\rangle$ and for the second moment $\left\langle\left[a^{\dagger}(t) a(t)\right]^{2}\right\rangle$ for different initial distributions. The first and second moments are used to get the output variances which are compared to those at the input in order to show the effect of the amplifier on the statistical properties of the radiation.

\section{QUANTUM MECHANICAL MODEL OF PARAMETRIC PROCESSES}

We are interested in obtaining a very simple quantum mechanical model for parametric interactions in order to evaluate the effect of the measuring (amplifying) process on the statistical properties of the radiation. We shall pick a model which is approximately equivalent to the simplest classical model for parametric interactions, viz., two lossless resonant circuits ${ }^{8}$ coupled by a time-varying reactance.

Our model consists of a cavity with perfectly conducting walls at $0^{\circ} \mathrm{K}$ which is designed to support an infinite number of nondegenerate modes. Parametric coupling among these modes is provided by modulating the dielectric constant (or permeability). Since, in general, an infinite number of modes will be coupled, the cavity, for simplicity, should be designed and the

\footnotetext{
${ }^{8}$ H. Suhl, Phys. Rev. 106, 384 (1957).
} 
dielectric constant variation must be chosen so that only two modes are coupled. Although this may present difficult design problems, they can be solved. ${ }^{9}$ Since all the important classical results may be obtained when only two modes are coupled, ${ }^{8,10}$ such a simplification loses no generality for our present purposes.

In order to consider small signal effects, the cavity modes are quantized by the familiar technique of field quantization ${ }^{11}$ and the fields are expressed in terms of creation and annihilation operators. The pump (dielectric constant) modulation is not quantized since it is assumed, as in the classical case, that the pump power is so large compared with the power in the cavity modes which are coupled that pump quantum fluctuations will be insignificant.

\section{QUANTIZATION OF THE CAVITY MODES}

Consider now a cavity with perfectly conducting walls at $0^{\circ} \mathrm{K}$. The field may be described classically in terms of a vector potential $\mathbf{A}(\mathbf{r}, t)$ where $\mathbf{r}$ is the position vector. The electric and magnetic fields are given by

$$
\mathbf{E}=-\frac{1}{c} \frac{\partial \mathbf{A}}{\partial t}, \quad \mathbf{H}=\operatorname{curl} \mathbf{A} .
$$

The vector potential may be expanded in a complete set of cavity modes by

$$
\mathbf{A}(\mathbf{r}, t)=\sum_{l} q_{l}(t) \mathbf{u}_{l}(\mathbf{r}),
$$

where the $q_{l}(t)$ satisfy

$$
d^{2} q_{l} / d t^{2}+\omega_{l}^{2} q_{l}=0
$$

and the normal modes, $\mathbf{u}_{l}$, satisfy

$$
\operatorname{curl} \operatorname{curl} \mathbf{u}_{l}=\left(\omega_{l} / c\right)^{2} \mathbf{u}_{l},
$$

subject to the boundary conditions at the cavity walls that the tangential components of $\mathbf{u}_{l}$ vanish as well as the normal components of $\operatorname{curl} \mathbf{u}_{l}$. We also normalize these modes so that

$$
\int_{\text {cavity }} \mathbf{u}_{l} \cdot \mathbf{u}_{m} d V=4 \pi c^{2} \delta_{l m} .
$$

The total energy of the field is

$$
H_{0}=\frac{1}{8 \pi} \int_{\text {cavity }}\left(\mathbf{E}^{2}+\mathbf{H}^{2}\right) d V .
$$

Now substituting for $\mathbf{E}$ and $\mathbf{H}$ in terms of $\mathbf{A}, \mathbf{E q}$. (3), using Eqs. (5), (6) as well as the vector identity

$$
(\operatorname{curl} \mathbf{u})^{2}=\operatorname{div}(\mathbf{u} \times \operatorname{curl} \mathbf{u})+\mathbf{u} \cdot \operatorname{curl} \operatorname{curl} \mathbf{u},
$$

\footnotetext{
${ }^{9}$ K. M. Poole and P. K. Tien, Proc. Inst. Radio Engrs. 46, 1387 (1958).

${ }_{10}$ P. K. Tien, J. Appl. Phys. 29, 1347 (1958).

${ }^{11}$ L. I. Schiff, Quantum Mechanics (McGraw-Hill Book Company, Inc., New York, 1949), First ed., Chapter XIV.
}

and the boundary conditions on the $\mathbf{u}_{l}$ 's, the field Hamiltonian reduces to

where

$$
H_{0}=\frac{1}{2} \sum_{l}\left[p_{l}^{2}+\omega_{l}^{2} q l^{2}\right]
$$

$$
d q_{l} / d t=p_{l}
$$

We now quantize by regarding the $p_{l}$ and $q_{l}$ as Hermitian operators satisfying the commutation relations

$$
\left[p_{l}, p_{m}\right]=\left[q_{l}, q_{m}\right]=0 ; \quad\left[q_{l}, p_{m}\right]=i \hbar \delta_{l m} .
$$

The operators $p_{l}$ and $q_{l}$ may be expressed in terms of non-Hermitian operators $a_{l}^{\dagger}$ and $a_{l}$ by means of

$$
\begin{aligned}
& q_{l}(t)=\left(\hbar / 2 \omega_{l}\right)^{\frac{1}{2}}\left[a_{l}^{\dagger}(t)+a_{l}(t)\right], \\
& p_{l}(t)=i\left(\hbar \omega_{l} / 2\right)^{\frac{1}{2}}\left[a_{l}^{\dagger}(t)-a_{l}(t)\right],
\end{aligned}
$$

where $a_{l}^{\dagger}$ is the Hermitian conjugate of $a_{l}$. The $a$ 's obey the commutation relations

$$
\begin{aligned}
{\left[a_{l}(t), a_{m}^{\dagger}(t)\right] } & =\delta_{l m} ; \\
{\left[a_{l}(t), a_{m}(t)\right] } & =\left[a_{l}^{\dagger}(t), a_{m}^{\dagger}(t)\right]=0 .
\end{aligned}
$$

In terms of the $a$ 's, the Hamiltonian (7) becomes

$$
H_{0}=\sum_{l} \hbar \omega_{l}\left[a_{l}^{\dagger} a_{l}+\frac{1}{2}\right] \equiv \sum_{l} H_{l} .
$$

The operators $a_{l}^{\dagger}(t)$ and $a_{l}(t)$ are the time-dependent Heisenberg creation and annihilation operators, respectively, for photons at $\omega_{l}$. An eigenstate of $a_{l}^{\dagger} a_{l}$ at $t=0$ may be specified by

$$
a_{l 0}^{\dagger} a_{l 0}\left|n_{l 0}\right\rangle=n_{l 0}\left|n_{l 0}\right\rangle
$$

where $n_{l 0}$ is the number of quanta at $\omega_{l}$ in the field and $\left|n_{l 0}\right\rangle$ is the wave function describing this state. This representation makes $H_{0}$ diagonal. Also

$$
\begin{aligned}
a_{l 0}^{\dagger}\left|n_{l 0}\right\rangle & =\left(n_{l 0}+1\right)^{\frac{1}{2}}\left|n_{l 0}+1\right\rangle, \\
a_{l 0}\left|n_{l 0}\right\rangle & =\left(n_{l 0}\right)^{\frac{1}{2}}\left|n_{l 0}-1\right\rangle,
\end{aligned}
$$

which show that $a^{\dagger}$ and $a$ are creation and annihilation operators.

The operators $a_{l}, a_{l}^{\dagger}$, and $H_{0}$ are in the Heisenberg representation. The Heisenberg equations of motion are

$$
\begin{aligned}
i \hbar d a_{l} / d t & =\left[a_{l}, H_{0}\right]=\hbar \omega_{l} a_{l}, \\
i \hbar d a_{l}^{\dagger} / d t & =\left[a_{l}^{\dagger}, H_{0}\right]=-\hbar \omega_{l} a_{l}^{\dagger},
\end{aligned}
$$

where the commutation relations (10) were used. The solutions are easily seen to be $a_{l}(t)=a_{l 0} \exp \left(-i \omega_{l} t\right)$ and $a_{l}^{\dagger}(t)=a_{l 0}^{\dagger} \exp \left(i \omega_{l} t\right)$. Thus, if initially the system is characterized by a state in which there are $n_{l}$ quanta at $\omega_{l}$, the state will not change with time.

\section{INTERACTION}

In order to provide coupling among the various cavity modes, we assume that the dielectric constant varies as

$$
\epsilon(\mathbf{r}, t)=1+\epsilon^{\prime}(\mathbf{r}, t) \equiv 1+\Delta \epsilon \cos (\omega t+\varphi) f(\mathbf{r}),
$$

where $f(\mathbf{r})$ is a function to be specified later. The weak 
coupling approximation assumes that $\Delta \epsilon \ll 1 . \varphi$ is an arbitrary pump phase.

The Hamiltonian for the system is now given by

$$
\begin{aligned}
H & =\frac{1}{8 \pi} \int_{\text {cavity }}\left[\epsilon(\mathbf{r}, t) \mathbf{E}^{2}+\mathbf{H}^{2}\right] d V \\
& =\frac{1}{8 \pi} \int_{\text {cavity }}\left[\mathbf{E}^{2}+\mathbf{H}^{2}\right] d V+\frac{1}{8 \pi} \int_{\text {cavity }} \epsilon^{\prime}(\mathbf{r}, t) \mathbf{E}^{2} d V .
\end{aligned}
$$

The first term is the unperturbed Hamiltonian of the previous section while the second term gives the coupling. If we express $\mathbf{E}$ and $\mathbf{H}$ in terms of the unperturbed cavity modes of the previous section, we can write the Hamiltonian as

$$
H=H_{0}+H^{\prime} \text {, }
$$

where $H_{0}$ is given by Eq. (11) and $H^{\prime}$ is

$$
H^{\prime}(t)=-\hbar \cos (\omega t+\varphi) \sum_{l, m} \kappa_{l m}\left(a_{l} \dagger-a_{l}\right)\left(a_{m}^{\dagger}-a_{m}\right),
$$

where Eq. (14) was used and the coupling coefficients are

$$
\kappa_{l m}=\kappa_{m l}=\frac{\Delta \epsilon}{16 \pi c^{2}}\left(\omega_{l} \omega_{m}\right)^{\frac{1}{2}} \int_{\text {cavity }} f(\mathbf{r}) \mathbf{u}_{l} \cdot \mathbf{u}_{m} d V .
$$

The Heisenberg equations of motion for the creation operators are given by Eq. (13) with $H_{0}$ replaced by $H_{0}+H^{\prime}$ which, on using the commutation relations (10), become

$$
\begin{aligned}
d a_{j}^{\dagger} / d t=i \omega_{j} a_{j}^{\dagger}+i\left[e^{i(\omega t+\varphi)}\right. & \\
& \left.+e^{-i(\omega t+\varphi)}\right] \sum_{l} \kappa_{j l}\left(a_{l}^{\dagger}-a_{l}\right),
\end{aligned}
$$

together with the Hermitian conjugate of these equations for $a_{j}$. Now in order that any modes be coupled, it is necessary to choose $f(\mathbf{r})$ so that the $\kappa_{j l} \neq 0$. In general, a given choice of $f(\mathbf{r})$ will leave an infinite number of modes coupled. As already noted, classical models for parametric interactions ${ }^{8,10}$ consider only a small number of interacting modes, usually only two, so that at first sight it would seem that our quantum model is not consistent with the classical one. By the use of a perturbation theory argument we can show that by the proper choice of the pump frequency $\omega$ we may limit the number of interacting modes to two.

In the absence of interaction $\left(\kappa_{j l}=0\right)$ the mode amplitudes are given by

$$
a_{s}^{\dagger}=a_{s 0}^{\dagger} e^{i \omega s t} ; \quad a_{s}=a_{s 0} e^{-i \omega_{s} t},
$$

so that to first order in the coupling coefficients, the contribution of $H^{\prime}(t)$ to $a_{j}^{\dagger}$ is given by

$$
\begin{aligned}
\left(\frac{d a_{j}^{\dagger}}{d t}\right)_{H^{\prime}}=i\left[e^{i(\omega t+\varphi)}+\right. & \left.e^{-i(\omega t+\varphi)}\right] \\
& \times \sum_{l} \kappa_{j l}\left[a_{l 0}^{\dagger} e^{i \omega l t}-a_{l 0} e^{-i \omega l t}\right] .
\end{aligned}
$$

Considering an arbitrary term $l=k$ on the right side of
(18), it is seen to contain frequency components at $\pm \omega \pm \omega_{k}$. For a continuous interaction between modes $j$ and $k$, we must fulfill the conditions $\omega_{j}=+\omega \pm \omega_{k}$ when $\omega>\omega_{k}$ or $\omega_{j}=\omega_{k} \pm \omega$ when $\omega_{k}>\omega$, so that the interaction gives rise to components varying as $\exp \left(i \omega_{j} t\right)$ which are synchronous with $a_{j}^{\dagger}(t)$. A mode $r$ for which the frequency conditions are not satisfied will give rise to components at frequencies $\left|\omega \pm \omega_{r}\right| \neq \omega_{j}$. The resultant beating at all the difference frequencies involved averages out to zero over time intervals long compared to the longest beating period.

As is well known from time-dependent perturbation theory, ${ }^{12}$ transitions which do not "conserve" energy must occur rapidly so that the energy deficiency $\Delta E$ and the transition period $\Delta t$ obey the uncertainty relation $\Delta E \Delta t \geq \hbar$. Consistent with this point of view it is clear that the neglect of the rapidly fluctuating nonsynchronous terms is identical to stating that they arise from the nonsecular terms of the Hamiltonian. This becomes clear on examination of Eq. (15) and considering final and initial states connected by the various terms and their total energy.

Barring accidental degeneracies, we therefore assume that only two nondegenerate modes are coupled by the pump field at $\omega$ and denotes them as the signal mode (1) and the idler mode (2). For $\omega=\omega_{1}+\omega_{2}$, Eq. (15) reduces to

$$
H_{\mathrm{amp}}{ }^{\prime}=-\hbar_{\kappa}\left[a_{1}^{\dagger} a_{2}^{\dagger} e^{-i(\omega t+\varphi)}+a_{1} a_{2} e^{i(\omega t+\varphi)}\right],
$$

and Eqs. (17) reduce to

$$
\begin{aligned}
& d a_{2}^{\dagger} / d t=i \omega_{2} a_{2}^{\dagger}-i \kappa e^{i(\omega t+\varphi)} a_{1}, \\
& d a_{1}^{\dagger} / d t=i \omega_{1} a_{1}^{\dagger}-i \kappa e^{i(\omega t+\varphi)} a_{2},
\end{aligned}
$$

plus their Hermitian conjugates. These terms arise from (19a) for which an equal number of signal and idler photons are created (or annihilated) simultaneously at the expense of the annihilation (or creation) of an equal number of pump photons. This situation gives rise, as will be shown in the next section, to amplification.

For a pump field satisfying $\omega=\omega_{1}-\omega_{2}$, Eq. (15) reduces to

$$
H_{\mathrm{conv}}{ }^{\prime}=+\hbar_{\kappa}\left[a_{1} a_{2}^{\dagger} e^{i(\omega t+\varphi)}+a_{1}^{\dagger} a_{2} e^{-i(\omega t+\varphi)}\right],
$$

and Eqs. (17) reduce to

$$
\begin{aligned}
& d a_{1} / d t=-i \omega_{1} a_{1}-i \kappa e^{-i(\omega t+\varphi)} a_{2}, \\
& d a_{2} / d t=-i \omega_{2} a_{2}-i \kappa e^{i(\omega t+\varphi)} a_{1},
\end{aligned}
$$

and their Hermitian conjugates. These terms arise from (19b) which correspond to transfer of energy between the signal and idler fields, i.e., frequency conversion, as will be shown later. We may add here that in order to show that the terms retained in (19a) and (19b) are secular, we must consider initial and final states which include the quantized pump field (excited to a high level).

\footnotetext{
${ }^{12}$ See reference 11 , Chapter 8 .
} 


\section{AMPLIFIER EQUATIONS OF MOTION}

The equations of motion for the creation and annihilation operators at $\omega_{1}$ and $\omega_{2}$ are [from Eq. (20)]

$$
\begin{aligned}
d a_{1} / d t & =-i \omega_{1} a_{1}+i \kappa e^{-i(\omega t+\varphi)} a_{2}{ }^{\dagger}, \\
d a_{2}{ }^{\dagger} / d t & =i \omega_{2} a_{2}{ }^{\dagger}-i \kappa e^{i(\omega t+\varphi)} a_{1},
\end{aligned}
$$

together with the Hermitian conjugates of these equations.

These equations will be immediately recognized as identical with the classical parametric amplifier equations $^{13}$ if the creation and annihilation operators are identified with the classical mode amplitudes and their complex conjugates, respectively. ( $\hbar$ has disappeared from the operator equations so this result is not too surprising.)

The Manley-Rowe relations ${ }^{13}$ follow directly from Eqs. (22) just as in the classical case since

$$
\frac{d}{d t} a_{1}^{\dagger} a_{1}=\frac{d}{d t} a_{2}^{\dagger} a_{2},
$$

which is equivalent to the commutator relations

$$
\left[a_{1}^{\dagger} a_{1}, H\right]=\left[a_{2}^{\dagger} a_{2}, H\right] \text {. }
$$

Thus, if $\bar{n}_{1}(t)$ and $\bar{n}_{2}(t)$ are the expectation values of $a_{1}^{\dagger} a_{1}$ and $a_{2}^{\dagger} a_{2}$, the number of photons at $\omega_{1}$ and $\omega_{2}$ at time $t$, it follows from Eq. (23) that the Manley-Rowe relations are equivalent to

$$
n_{1}(t)-n_{1}(0)=n_{2}(t)-n_{2}(0),
$$

showing that each time the pump creates a photon at $\omega_{1}$ it must also create one at $\omega_{2}$ which again is a necessary consequence of energy conservation as already noted. ${ }^{14}$ Energy conservation therefore dictates the nature of the coupling mechanism between the creation and annihilation operators.

The solutions of the equations of motion (22) are easily found to be

$$
\begin{aligned}
a_{1}(t) & =e^{-i \omega_{1} t}\left\{a_{10} \cosh \kappa t+i e^{-i \varphi} a_{20}{ }^{\dagger} \sinh \kappa t\right\}, \\
a_{2}{ }^{\dagger}(t) & =e^{i \omega_{2} t}\left\{a_{20}{ }^{\dagger} \cosh \kappa t-i e^{i \varphi} a_{10} \sinh \kappa t\right\},
\end{aligned}
$$

with their Hermitian conjugates. The subscript zero refers to initial values. From these equations it follows that

$$
\begin{aligned}
a_{1}^{\dagger} a_{1}=a_{10}{ }^{\dagger} a_{10} & \cosh ^{2} \kappa t+\left(1+a_{20}^{\dagger} a_{20}\right) \sinh ^{2} \kappa t \\
& +\frac{1}{2} i \sinh 2 \kappa t\left[a_{10}{ }^{\dagger} a_{20}{ }^{\dagger} e^{-i \varphi}-a_{10} a_{20} e^{i \varphi}\right], \\
a_{2}^{\dagger} a_{2}=a_{20}{ }^{\dagger} a_{20} & \cosh ^{2} \kappa t+\left(1+a_{10}{ }^{\dagger} a_{10}\right) \sinh ^{2} \kappa t \\
& +\frac{1}{2} i \sinh 2 \kappa t\left[a_{10}{ }^{\dagger} a_{20} e^{-i \varphi}-a_{10} a_{20} e^{i \varphi}\right] .
\end{aligned}
$$

${ }^{13}$ W. H. Louisell, Coupled Mode and Parametric Electronics (John Wiley \& Sons, Inc., New York, 1960), 100; 119; 104; 96.

${ }^{14} \mathrm{It}$ has been pointed out by M. Weiss [Quantum ElectronicsA Symposium, edited by C. H. Townes (Columbia University Press, New York, 1960), p. 291], by J. R. Pierce [J. Appl. Phys. 30, 1341 (1959)] and by others that the $\beta$ conditions are equivalent to the conservation of momentum in a dispersive medium while the conservation of quanta is equivalent to the Manley-Rowe relations.
One now sees from these equations how the operators differ from the classical mode amplitudes. The noncommutivity of $a_{1}$ and $a_{1}^{\dagger}$ as well as $a_{2}$ and ${a_{2}}^{\dagger}$ lead to an extra 1 in the second term of Eqs. (26) and (27). If $a_{20}{ }^{\dagger} a_{20}=n_{20}$ are the initial number of photons at the idler and $a_{10}{ }^{\dagger} a_{10}=n_{10}$ the initial number at the signal, then even if $n_{10}=n_{20}=0$, after a time $t$ there will be photons at $\omega_{1}$ and $\omega_{2}$ which can be viewed as arising from the zero-point fluctuations of the signal and idler fields or equivalently as due to our inability to specify the initial number of photons more accurately.

For future reference the displacement coordinates and momenta [Eq. (9)] are given:

$$
\begin{gathered}
p_{1}(t)=i\left(\hbar \omega_{1} / 2\right)^{\frac{1}{2}}\left[\cosh \kappa t\left(a_{10}^{\dagger} e^{i \omega_{1} t}-a_{10} e^{-i \omega_{1} t}\right)\right. \\
\left.-i \sinh \kappa t\left(a_{20} e^{i\left(\omega_{1} t+\varphi\right)}+a_{20} e^{\dagger} e^{-i\left(\omega_{1} t+\varphi\right)}\right)\right], \\
q_{1}(t)=\left(\hbar / 2 \omega_{1}\right)^{\frac{1}{2}}\left[\cosh \kappa t\left(a_{10} e^{i \omega_{1} t}+a_{10} e^{-i \omega_{1} t}\right)\right. \\
\left.-i \sinh \kappa t\left(a_{20} e^{i\left(\omega_{1} t+\varphi\right)}-a_{20} e^{-i\left(\omega_{1} t+\varphi\right)}\right)\right],
\end{gathered}
$$

with similar expressions for $p_{2}(t)$ and $q_{2}(t)$ which can be obtained from the above expressions with subscripts 1 and 2 interchanged everywhere. We shall use these expressions later to evaluate the uncertainty in the number of quanta and the phase of the signal and idler modes.

\section{EXPECTATION VALUES AND FLUCTUATIONS}

We are now interested in considering the fluctuations in the output of the parametric amplifier. These fluctuations will determine the accuracy with which we can measure the number of photons in the input wave by an examination of the output wave. To study these fluctuations under large amplification we must obtain expectation values of the number of quanta at the output.

We will first focus our attention on the average number of $\omega_{1}$ photons, $\bar{n}_{1}(t)$, and the $\omega_{2}$ photons, $\bar{n}_{2}(t)$, both as a function of time. We assume that at $t=0$ there are exactly $n_{10}$ of the $\omega_{1}$ photons and $n_{20}$ photons at frequency $\omega_{2}$. This is equivalent to taking as our wave functional at $t=0$

$$
\begin{aligned}
& \psi(0)=\left|n_{10}, n_{20}\right\rangle, \\
\bar{n}_{1}(t) \text { is given by } & \\
\bar{n}_{1}(t)= & \left\langle a_{1}^{\dagger}(t) a_{1}(t)\right\rangle \\
= & \left\langle n_{10}, n_{20}\right| a_{10}{ }^{\dagger} a_{10} \cosh ^{2} \kappa t+\left(1+a_{20}{ }^{\dagger} a_{20}\right) \sinh ^{2} \kappa t \\
& +\frac{1}{2} i \sinh ^{2} \kappa t\left(a_{10}{ }^{\dagger} a_{20}{ }^{\dagger} e^{-i \varphi}-a_{10} a_{20} e^{+i \varphi}\right)\left|n_{10}, n_{20}\right\rangle \\
= & n_{10} \cosh ^{2} \kappa t+\left(1+n_{20}\right) \sinh ^{2} \kappa t,
\end{aligned}
$$

where we made use of Eq. (12). For large gain we have

$$
\bar{n}_{1}(t)=K\left(n_{10}+n_{20}+1\right),
$$

where $K \approx \exp (2 \kappa t) / 4$ is the gain. A similar procedure leads to

$$
\bar{n}_{2}(t)=\left\langle a_{2}^{\dagger}(t) a_{2}(t)\right\rangle=n_{20} \cosh ^{2} \kappa t+\left(1+n_{10}\right) \sinh ^{2} \kappa t .
$$

We may consider the variable $t$ as the time variable 
in a resonant (cavity) type of an amplifier or, equivalently, as the distance along a distributed amplifier so that $\cosh ^{2} \kappa t \gg 1$ corresponds to a high gain amplifier. Equation (30) or (31) is identical, with one exception, to the classical expression for the power along the distributed parametric amplifier. ${ }^{10}$ The classical expression has $n_{20}$ in the second term on the right side of Eq. (30) instead of $\left(1+n_{20}\right)$ in our case. The added term $\sinh ^{2} \kappa t$ corresponds to an output whose magnitude is independent of the input and constitutes the noncoherent zero-point fluctuation noise. The same remark applies to Eq. (31). The Manley-Rowe relations are fulfilled since

$$
\bar{n}_{1}(t)-n_{10}=\bar{n}_{2}(t)-n_{20}
$$

Since the number of photons $n$ is related to the power $P$ by $n=P / \hbar \omega$ we can rewrite Eq. (32) as

$$
P_{1} / \omega_{1}=P_{2} / \omega_{2}
$$

in which form it was originally given by Manley and Rowe. ${ }^{13}$

For a measure of the output fluctuations we compute the output variance $\left(\Delta n_{1}\right)^{2}$ given by

where

$$
\left(\Delta n_{1}\right)^{2}=\left\langle\left(n_{1}-\bar{n}_{1}\right)^{2}\right\rangle_{\mathrm{av}}=\left\langle n_{1}^{2}\right\rangle_{\mathrm{av}}-\bar{n}_{1}^{2},
$$

$$
\begin{aligned}
\left\langle n_{1}^{2}\right\rangle_{\mathrm{av}}= & \left\langle n_{10}, n_{20}\left|\left(a_{1}^{\dagger} a_{1}\right)^{2}\right| n_{10}, n_{20}\right\rangle \\
= & n_{10}{ }^{2} \cosh ^{4} \kappa t+2 n_{10}\left(1+n_{20}\right) \cosh ^{2} \kappa t \sinh ^{2} \kappa t \\
& +\left(1+n_{20}\right)^{2} \sinh ^{4} \kappa t \\
& \quad+\frac{1}{4} \sinh ^{2} \kappa t\left[2 n_{10} n_{20}+n_{10}+n_{20}+1\right] .
\end{aligned}
$$

Using Eq. (30) for $\bar{n}_{1}$ the variance $\left(\Delta n_{1}\right)^{2}$ takes the form

$$
\begin{aligned}
\left(\Delta n_{1}\right)^{2} & =\frac{1}{4} \sinh 2 \kappa t\left(1+n_{10}+n_{20}+2 n_{10} n_{20}\right) \\
& \approx K^{2}\left(1+n_{10}+n_{20}+2 n_{10} n_{20}\right),
\end{aligned}
$$

where the approximate equality stands, as will be the case in the remainder of this paper, for the high-gain case : $K \gg 1$.

It should be noted that although the input variance is zero, if we have to deduce the input variance from an examination of the output we will get a finite result in accordance with Eq. (36a). This represents the uncertainty introduced by the amplifying mechanism itself.

The fractional variance $\mathcal{E}^{2}$ is defined by

$$
\mathcal{E}^{2}=\left(\Delta n_{1}\right)^{2} /\left(\bar{n}_{1}\right)^{2},
$$

and for $K \gg 1$ is given by

$$
\mathcal{E}^{2} \approx\left(1+n_{10}+n_{20}+2 n_{10} n_{20}\right) /\left(1+n_{10}+n_{20}\right)^{2} .
$$

For single frequency input we may set $n_{20}=0$ and $\mathcal{E}^{2}$ becomes

$$
\mathcal{E}^{2} \approx 1 /\left(1+n_{10}\right),
$$

which is identical with the result obtained by Shimoda et $a l .{ }^{1}$ for the ideal maser amplifier.

The preceding treatment, which assumed an exact knowledge of the number of input photons $n_{10}$ and $n_{20}$, is not always satisfactory. It entails, in accordance with the uncertainty principle, a complete abandonment of any phase information about the incoming waves. This model does not reflect the fact that the very process which causes our signal to be weak introduces a fluctuation in the number of photons. The most common cause for the signal weakness is the distance between the signal source and the amplifier (the receiver) which, because of the small probability that a given emitted photon will arrive at the receiver, leads to a Poisson distribution at the input. If the signal weakness is due to lossy attenuation the resulting distribution at the input to the amplifier is again Poisson $^{6}$ and is due to the random nature of the absorption.

Both the phase information and the Poisson nature of the distribution are included if the wave functionals $|\psi(0)\rangle$ are taken as a Poisson distribution over the states of the uncoupled system at $t=0$, before amplification begins. ${ }^{15}$ (See Appendix I.)

$$
|\psi(0)\rangle=\sum_{n_{10}, n_{20}}\left[p\left(n_{10}\right) p\left(n_{20}\right)\right]^{\frac{1}{2}} e^{-i\left(n_{10} \varphi_{1}+n_{20} \varphi_{2}\right)}\left|n_{10}, n_{20}\right\rangle .
$$

Here $\varphi_{1}$ and $\varphi_{2}$ are the phases of the signal and idler waves, and the $p\left(n_{i 0}\right)$ are the Poisson probability distribution functions

$$
p\left(n_{i 0}\right)=\frac{\exp \left(-n_{i 0}\right) \bar{n}_{i 0} n_{i 0}}{\left(n_{i 0}\right) !} \quad(i=1,2)
$$

where $\bar{n}_{i 0}$ is the average number of photons at $\omega_{i}$ at $t=0$. That Eq. (39) does actually correspond to a definite phase can be illustrated by calculating the expectation value for the electric or magnetic field.

A few expectation values which are needed in the calculation of the first and second moments of the output are

$$
\begin{aligned}
\left\langle a_{1}^{\dagger} a_{1}\right\rangle= & \bar{n}_{1}(t)=\bar{n}_{10} \cosh ^{2} \kappa t+\left(1+\bar{n}_{20}\right) \sinh ^{2} \kappa t \\
& -\sinh 2 \kappa t\left(\left\langle n_{10} n_{20}\right\rangle_{\mathrm{av}}\right)^{\frac{1}{2}} \sin \left(\varphi_{1}+\varphi_{2}-\varphi\right), \\
\left\langle a_{2}^{\dagger} a_{2}\right\rangle= & \bar{n}_{2}(t)=\bar{n}_{20} \cosh ^{2} \kappa t+\left(1+\bar{n}_{10}\right) \sinh ^{2} \kappa t \\
& -\sinh 2 \kappa t\left(\left\langle n_{10} n_{20}\right\rangle_{\mathrm{av}}\right)^{\frac{1}{2}} \sin \left(\varphi_{1}+\varphi_{2}-\varphi\right), \\
\left\langle\left({a_{1}}^{\dagger} a_{1}\right)^{2}\right\rangle= & \left\langle n_{1}^{2}(t)\right\rangle_{\mathrm{av}}=\left\langle n_{10}\right\rangle_{\mathrm{av}} \cosh ^{4} \kappa t \\
& +\left(1+2 \bar{n}_{20}+\left\langle n_{20}{ }^{2}\right\rangle_{\mathrm{av}}\right) \sinh ^{4} \kappa t \\
& +\left[1+3 \bar{n}_{10}+\bar{n}_{20}+4\left\langle n_{10} n_{20}\right\rangle_{\mathrm{av}}\right. \\
& \left.-2\left\langle n_{10} n_{20}\right\rangle_{\mathrm{av}} \cos 2\left(\varphi_{1}+\varphi_{2}-\varphi\right)\right] \sinh ^{2} \kappa t \\
& \times \cosh ^{2} \kappa t-4\left(\left\langle n_{10} n_{20}\right\rangle_{\mathrm{av}}\right)^{\frac{1}{2}} \sin \left(\varphi_{1}+\varphi_{2}-\varphi\right) \\
& \times\left[\left(1+\bar{n}_{10}\right) \sinh \kappa t \cosh ^{3} \kappa t+\left(1+\bar{n}_{20}\right)\right. \\
& \left.\quad \times \sinh ^{3} \kappa t \cosh \kappa t-\frac{1}{2} \sinh \kappa t \cosh \kappa t\right] .
\end{aligned}
$$

A typical calculation used to derive Eq. (43) will be shown in Appendix II. For the large gain case, $K \gg 1$,

15 I. R. Senitzky, Phys. Rev. 95, 904 (1954). 
we may use

$$
\begin{aligned}
\bar{n}_{1}(t) \approx & {\left[1+\bar{n}_{10}+\bar{n}_{20}\right.} \\
& \left.-2\left(\left\langle n_{10} n_{20}\right\rangle_{\mathrm{av}}\right)^{\frac{1}{2}} \sin \left(\varphi_{1}+\varphi_{2}-\varphi\right)\right] K, \\
\left\langle n_{1}{ }^{2}(t)\right\rangle_{\mathrm{av}} \approx & \left\{\left\langle n_{10}{ }^{2}\right\rangle_{\mathrm{av}}+\left\langle n_{20}{ }^{2}\right\rangle_{\mathrm{av}}+3\left(\bar{n}_{10}+\bar{n}_{20}\right)+2\right. \\
& +4\left\langle n_{10} n_{20}\right\rangle_{\mathrm{av}}-2\left\langle n_{10} n_{20}\right\rangle_{\mathrm{av}} \cos 2\left(\varphi_{1}+\varphi_{2}-\varphi\right) \\
& -4\left(\left\langle n_{10} n_{20}\right\rangle_{\mathrm{av}}\right)^{\frac{1}{2}} \sin \left(\varphi_{1}+\varphi_{2}-\varphi\right) \\
& \left.\times\left[\bar{n}_{10}+\bar{n}_{20}+2\right]\right\} K^{2} .
\end{aligned}
$$

The output variance is then given by

$$
\begin{aligned}
\left(\Delta n_{1}\right)^{2}= & \left\langle n_{1}{ }^{2}\right\rangle_{\mathrm{av}}-\bar{n}_{1}{ }^{2} \\
= & K^{2}\left\{1+2\left(\bar{n}_{10}+\bar{n}_{20}\right)\right. \\
& \left.\quad-4\left(\left\langle n_{10} n_{20}\right\rangle_{\mathrm{av}}\right)^{\frac{1}{2}} \sin \left(\varphi_{1}+\varphi_{2}-\varphi\right)\right\} .
\end{aligned}
$$

For a single frequency input, $\tilde{n}_{20}=0$, we get

$$
\left(\Delta n_{1}\right)^{2}=K^{2}\left(1+2 \bar{n}_{10}\right),
$$

which is to be compared to the value

$$
\left(\Delta n_{1}\right)^{2}=K^{2}\left(1+\bar{n}_{10}\right),
$$

obtained for the case when the number of input photons was perfectly well defined [Eq. (36a) $]$ for $n_{20}=0$. The effect of the parametric amplification in both cases is to increase the variance of the output (divided by $K^{2}$ ) over that of the input by $\left(1+n_{10}\right)$.

\section{NOISE IN PARAMETRIC AMPLIFIERS}

The output noise power at an amplifier is usually defined as the average output noise power measured in the absence of any input. Since the number of photons $\bar{n}_{1}$ and the average power $\bar{P}_{1}$ are related by

$$
\bar{P}_{1}=\bar{n}_{1} h \nu_{1} d \nu,
$$

for an amplifier with an effective bandwidth $d \nu$, the output power for the case of a Poisson distributed input is

$$
\begin{aligned}
\bar{P}_{1}=[1+ & \bar{n}_{10}+\bar{n}_{20} \\
& \left.-2\left(\left\langle n_{10} n_{20}\right\rangle_{\mathrm{av}}\right)^{\frac{1}{2}} \sin \left(\varphi_{1}+\varphi_{2}-\varphi\right)\right] K h \nu_{1} d \nu .
\end{aligned}
$$

The noise power is that part of $\bar{P}_{1}$ which does not contain either $\bar{n}_{10}$ or $\bar{n}_{20}$ and is given by

$$
\bar{P}_{N 1}=K h \nu_{1} d \nu \text {. }
$$

An equal amount of output power results when the input rate of photons is one photon per $(d \nu)^{-1}$ seconds. So that the limiting detecting sensitivity of the parametric amplifier is one photon per resolution time of the receiving system. The same results obtains for the ideal maser amplifier when most of the "spins" are in the excited level.

The "noisiness" of amplifiers is often described in terms of an "effective source temperature" $T_{e}$, which is the temperature of a matched input termination yielding an output noise power equal to that generated

${ }^{16}$ J. P. Gordon and L. D. White, Proc. Inst. Radio Engrs. 46, 1588 (1958). by the amplifier. Since the available power from a termination at temperature $T$ between $\omega$ and $\omega+d \omega$ is given by

$$
K \frac{\hbar \omega}{e^{\hbar \omega / k T}-1} d \omega
$$

we get by equating Eq. (51) to Eq. (50)

$$
T_{e}=\frac{\hbar \omega}{k} / \ln 2 .
$$

It should be noted that Eq. (52) or (50) give the contribution to the noise temperature which is basic to the parametric process. This is a limit which can only be approached by minimizing the noise contributing losses and by cooling temperatures comparable to $\hbar \omega / k$.

\section{COMPLEMENTARY}

It is next of interest to consider how well the number of photons in an incoming wave as well as the phase can be determined, simultaneously, by an examination of the output.

Under the assumption that the signal satisfies a Poisson distribution, we may calculate the expectation values of the canonically conjugate coordinates and momenta as given by Eqs. (28) and (29). These are found to be (for large gain)

$$
\begin{aligned}
\bar{p}_{1}=-\left(2 \hbar \omega_{1}\right)^{\frac{1}{2}}\left\{\left(\bar{n}_{10}\right)^{\frac{1}{2}}\right. & \sin \left(\omega_{1} t+\varphi_{1}\right) \\
& \left.-\left(\bar{n}_{20}\right)^{\frac{1}{2}} \cos \left(\omega_{1} t+\varphi-\varphi_{2}\right)\right\} K^{\frac{1}{2}}, \\
\bar{q}_{1}=\left(2 \hbar / \omega_{1}\right)^{\frac{1}{2}}\left\{\left(\bar{n}_{10}\right)^{\frac{1}{2}}\right. & \cos \left(\omega_{1} t+\varphi_{1}\right) \\
& \left.+\left(\bar{n}_{20}\right)^{\frac{1}{2}} \sin \left(\omega_{1} t+\varphi-\varphi_{2}\right)\right\} K^{\frac{1}{2}},
\end{aligned}
$$

$\bar{p}_{2}$ and $\bar{q}_{2}$ are found by replacing subscript 1 by 2 and 2 by 1 in the above.

Also the mean squares are

$$
\begin{aligned}
\left\langle p_{1}{ }^{2}\right\rangle_{\mathrm{av}}=\left(\hbar \omega_{1} / 2\right) & \left\{\left[1+4 \bar{n}_{10} \sin ^{2}\left(\omega_{1} t+\varphi_{1}\right)\right]\right. \\
& +\left[1+4 \bar{n}_{20} \cos ^{2}\left(\omega_{1} t+\varphi-\varphi_{2}\right)\right] \\
& -8\left(\left\langle n_{10} n_{20}\right\rangle_{\mathrm{av}}\right)^{\frac{1}{2}} \sin \left(\omega_{1} t+\varphi_{1}\right) \\
& \left.\times \cos \left(\omega_{1} t+\varphi-\varphi_{2}\right)\right\} K, \\
\left\langle q_{1}^{2}\right\rangle_{\mathrm{av}}=\left(\hbar / 2 \omega_{1}\right)\left\{\left[1+4 \bar{n}_{10} \cos ^{2}\left(\omega_{1} t+\varphi_{1}\right)\right]\right. & +\left[1+4 \bar{n}_{20} \sin ^{2}\left(\omega_{1} t+\varphi-\varphi_{2}\right)\right] \\
+ & +8\left(\left\langle n_{10} n_{20}\right\rangle_{\mathrm{av}}\right)^{\frac{1}{2}} \cos \left(\omega_{1} t+\varphi_{1}\right) \\
& \left.\times \sin \left(\omega_{1} t+\varphi-\varphi_{2}\right)\right\} K .
\end{aligned}
$$

while again $\left\langle p_{2}^{2}\right\rangle_{\text {av }}$ is obtained from $\left\langle p_{1}^{2}\right\rangle_{\text {av }}$ by interchanging 1 and 2 everywhere and $\left\langle q_{2}^{2}\right\rangle_{\text {av }}$ is obtained in the same manner from $\left\langle q_{1}{ }^{2}\right\rangle_{\text {av }}$.

The second moments at the output are given by

$$
\begin{aligned}
& \left(\Delta p_{1}\right)^{2}=\left\langle p_{1}{ }^{2}\right\rangle_{\mathrm{av}}-\bar{p}_{1}{ }^{2}=\hbar \omega_{1} K, \\
& \left(\Delta q_{1}\right)^{2}=\left\langle q_{1}{ }^{2}\right\rangle_{\mathrm{av}}-\bar{q}_{1}{ }^{2}=\left(\hbar / \omega_{1}\right) K, \\
& \left(\Delta p_{2}\right)^{2}=\hbar \omega_{2} K, \\
& \left(\Delta q_{2}\right)^{2}=\left(\hbar / \omega_{2}\right) K,
\end{aligned}
$$


and the phases of the signal, idler, and pump have disappeared. Thus the initial uncertainties in measuring the amplitudes of the fields are

$$
\Delta p_{10} \Delta q_{10}=\hbar, \quad \Delta p_{20} \Delta q_{20}=\hbar,
$$

where $\Delta p_{1}=K \Delta p_{10}, \Delta q_{1}=K \Delta q_{10}$, etc.

Note that the uncertainty product $\Delta p_{10} \Delta q_{10}$ is twice the minimum value. This can be traced to the two "1's" in Eq. (54), showing that the total fluctuation in either channel is due to equal contributions from both channels and that even when $n_{20}=0$, the zero-point vibrations of the idler channel introduce noise (fluctuations) into the signal channel.

Consider the case in which $\bar{n}_{20}=0$. Then by Eq. (53),

$$
\begin{aligned}
\bar{p}_{1} & =-\left(2 \hbar \omega_{1} \bar{n}_{10}\right)^{\frac{1}{2}}\left[\cos \varphi_{1} \sin \omega_{1} t+\sin \varphi_{1} \cos \omega_{1} t\right] K^{\frac{1}{2}} \\
& \equiv\left[p_{10} \cos \omega_{1} t-\omega_{1} q_{10} \sin \omega_{1} t\right] K^{\frac{1}{2}}, \\
\bar{q}_{1} & =\left(2 \hbar n_{10} / \omega_{1}\right)^{\frac{1}{2}}\left[\cos \varphi_{1} \cos \omega_{1} t-\sin \varphi_{1} \sin \omega_{1} t\right] K^{\frac{1}{2}} \\
& \equiv\left[q_{10} \cos \omega_{1} t+\left(p_{10} / \omega_{1}\right) \sin \omega_{1} t\right] K^{\frac{1}{2}} .
\end{aligned}
$$

Thus it follows that the phase $\varphi_{1}$ is

$$
\varphi_{1}=\tan ^{-1} \frac{p_{10}}{\omega_{1} q_{10}},
$$

or, by differentiation, the uncertainty in phase is

$$
\Delta \varphi_{1}=\frac{\omega_{1} q_{10} \Delta p_{10}-p_{10} \omega_{1} \Delta q_{10}}{\left(p_{10}{ }^{2}+\omega_{1}^{2} q_{10}{ }^{2}\right)}
$$

if the uncertainties, $\Delta p_{10}$ and $\Delta q_{10}$, are suitably small. If the fluctuations in $\Delta p_{10}$ and $\Delta q_{10}$ are uncorrelated, we have

$$
\left(\Delta \varphi_{1}\right)^{2}=\frac{\omega_{1}^{2} q_{10}{ }^{2}\left(\Delta p_{10}\right)^{2}+\omega_{1}^{2} p_{10}{ }^{2} \Delta q_{10}{ }^{2}}{\left(p_{10}{ }^{2}+\omega_{1}^{2} q_{10}\right)^{2}} .
$$

We have therefore been able to relate the uncertainty in the phase of the signal to uncertainties in the field amplitudes.

We have shown in Eq. (55)

so that

$$
\Delta p_{10}=\omega_{1} \Delta q_{10}
$$

Since by (57) and (58), $p_{10}{ }^{2}+\omega_{1}^{2} q_{10}{ }^{2}=2 \hbar \omega_{1} \bar{n}_{10}$. By Eq. (55)

$$
\left(\Delta q_{10}\right)^{2}=\hbar / \omega_{1} .
$$

Equation (63) becomes

By Eq. (47),

$$
\therefore \quad\left(\Delta \varphi_{1}\right) \cong 1 /\left(2 \bar{n}_{10}\right)^{\frac{1}{2}} .
$$

so that

$$
\Delta n_{1} / K=\Delta n_{10}{ }^{\prime}=\left(1+2 \bar{n}_{10}\right)^{\frac{1}{2}},
$$

$$
\Delta \varphi_{1} \Delta n_{10}{ }^{\prime}=\left[\left(2 \bar{n}_{10}+1\right) / 2 \bar{n}_{10}\right]^{\frac{1}{2}} .
$$

This result is identical with that found by Townes and
Serber $^{2}$ for the maser case except for a factor of 2 discussed in connection with Eq. (56).

\section{FREQUENCY CONVERTER}

The frequency converter can be treated by the same methods using Eq. (19b) as the interaction Hamiltonian. From the nature of this coupling which is determined from energy conservation between the pump, signal, and idler, viz., $\hbar \omega=\hbar \omega_{1}-\hbar \omega_{2}$, it follows that only annihilation operators are coupled to annihilation operators at the two frequencies. The equations of motion are given by Eq. (21):

$$
\begin{aligned}
& d a_{1} / d t=-i \omega_{1} a_{1}-i \kappa e^{-i(\omega t+\varphi)} a_{2}, \\
& d a_{2} / d t=-i \omega_{2} a_{2}-i \kappa e^{i(\omega t+\varphi)} a_{1},
\end{aligned}
$$

with the Hermitian conjugate of these equations in which only creation operators are coupled. These will again be recognized as the classical frequency converter equations ${ }^{13}$ where the $a$ 's are the mode amplitudes.

These equations can be solved to yield

$$
\begin{aligned}
& a_{1}^{\dagger} a_{1}=a_{10}{ }^{\dagger} a_{10} \cos ^{2} \kappa t+a_{20}{ }^{\dagger} a_{20} \sin ^{2} \kappa t \\
& +\frac{1}{2} i\left(a_{20}{ }^{\dagger} a_{10} e^{i \varphi}-a_{10}{ }^{\dagger} a_{20} e^{-i \varphi}\right) \sin 2 \kappa t,
\end{aligned}
$$

and

$$
\begin{aligned}
a_{2}^{\dagger} a_{2}=a_{20}{ }^{\dagger} a_{20} \cos ^{2} \kappa t+a_{10}^{\dagger} a_{10} \sin ^{2} \kappa t & \\
& -\frac{1}{2} i\left(a_{20}{ }^{\dagger} a_{10} e^{i \varphi}-a_{10}{ }^{\dagger} a_{20} e^{-i \varphi}\right) \sin 2 \kappa t .
\end{aligned}
$$

If at $t=0$, there are exactly $n_{10}$ photons at $\omega_{1}$ and $n_{20}$ photons at $\omega_{2}$ the solutions for the expectation values $\bar{n}_{1}(t)$ and $\bar{n}_{2}(t)$ are

$$
\begin{aligned}
& \bar{n}_{1}(t)=\left\langle a_{1}^{\dagger}(t) a_{1}(t)\right\rangle=n_{10} \cos ^{2} \kappa t+n_{20} \sin ^{2} \kappa t, \\
& \bar{n}_{2}(t)=\left\langle a_{2}^{\dagger}(t) a_{2}(t)\right\rangle=n_{20} \cos ^{2} \kappa t+n_{10} \sin ^{2} \kappa t .
\end{aligned}
$$

In a typical frequency conversion we have $n_{20}=0$, for which case Eqs. (70) and (71) become

$$
\begin{aligned}
& \bar{n}_{1}(t)=n_{10} \cos ^{2} \kappa t, \\
& \bar{n}_{2}(t)=n_{10} \sin ^{2} \kappa t,
\end{aligned}
$$

so that at $t=(\pi / 2 \kappa)(2 m+1)$, the input which was launched at frequency $\omega_{1}$ can be taken off at $\omega_{2}$. An interesting feature of the frequency converter is that, unlike the amplifier, it has no zero-point fluctuations in its output since, according to Eqs. (70) and (71), with $n_{10}=n_{20}=0$ there is no output.

The difference between the parametric amplifier and the frequency converter as regards their zero-point fluctuations can be traced to the basic quantum mechanical model. In the parametric amplifier a simultaneous generation of a signal $\left(\omega_{1}\right)$ photon and an idler $\left(\omega_{2}\right)$ photon corresponds to a transition $\left|n_{1}, n_{2}\right\rangle \rightarrow\left|n_{1}+1, n_{2}+1\right\rangle$ whose strength is proportional to $\left(n_{1}+1\right)\left(n_{2}+1\right)$ and which can, therefore, take place even when $n_{1}=n_{2}=0$. In the case at the frequency converter the mechanism of transfer of energy from $\omega_{2}$ to $\omega_{1}$ is described by a $\left|n_{1}, n_{2}\right\rangle \rightarrow\left|n_{1}+1, n_{2}-1\right\rangle$ 
process whose strength is proportional to $\left(n_{1}+1\right)\left(n_{2}\right)$ so that it cannot take place when the input, $n_{2}$, is zero.

\section{SUMMARY}

Four aspects of the parametric interactions have been investigated. First we establish the correspondence between the classical limit of our quantum mechanical model and the familiar, wholly classical, model. Secondly we showed the existence of zero-point fluctuations in the output of the parametric amplifier. We next showed that the phase and the number of quanta of an incoming electromagnetic wave can be measured with an accuracy limited only by the uncertainty principle. A brief treatment of the frequency converter shows the absence of zero-point fluctuations from its output.

\section{ACKNOWLEDGMENTS}

The authors are indebted to Dr. P. K. Tien and Dr. J. P. Gordon for several valuable discussions.

\section{APPENDIX I}

It will be shown here that a Poisson distribution over the energy eigenstates of the quantized field in a cavity oscillating at frequency $\omega$ lead to a minimum uncertainty wave packet. The electric and magnetic fields can then be specified to an ultimate precision limited by the uncertainty relations and the expectation values of the fields for the "Poisson-state" lead to the classical results. This is an example of Ehrenfest's theorem.

For simplicity, the electric and magnetic fields of the cavity are written as

$$
\mathbf{E}(\mathbf{r}, t)=-4 \pi c p(t) \mathbf{u}(\mathbf{r}), \quad \mathbf{H}(\mathbf{r}, t)=q(t) \operatorname{curl} \mathbf{u}(\mathbf{r}),
$$

were, as usual, $q$ and $p$ satisfy the commutation relations

$$
[q, p]=i \hbar ; \quad[q, q]=[p, p]=0 .
$$

The quantized field Hamiltonian is given by

$$
H=\left(\omega^{2} / 8 \pi c^{2}\right) q^{2}+2 \pi c^{2} p^{2} .
$$

In the momentum representation, $q=i \hbar \partial / \partial p$ and the Schrödinger equation becomes

where

$$
d^{2} u_{n} / d \xi^{2}+\left[\Lambda_{n}-\xi^{2}\right] u_{n}=0,
$$

$$
\xi=\alpha p ; \quad \alpha^{2}=4 \pi c^{2} / \hbar \omega ; \quad \Lambda_{n}=2 E_{n} / \hbar \omega=2 n+1,
$$

and

$$
u_{n}(\xi)=\left(\alpha / 2^{n} n ! \sqrt{ } \pi\right)^{\frac{1}{2}} \exp \left(-\xi^{2} / 2\right) H_{n}(\xi),
$$

where the $H_{n}(\xi)$ are Hermite polynomials.

$u_{n}$ is an energy eigenstate corresponding to $n$ photons in the radiation field at frequency $\omega$.

Now let us consider a linear combination of these eigenstates weighted with a Poisson distribution, viz.,

$$
\varphi(p, t)=\sum_{n=0}^{\infty} c_{n} e^{-i \omega t\left(n+\frac{1}{2}\right)} u_{n}(\alpha p),
$$

where

$$
c_{n}=\left(e^{-\lambda} \lambda^{n} / n !\right)^{\frac{1}{2}} e^{-i n \varphi},
$$

where $\lambda$ has the physical significance of the average number of quanta in the field as will be shown below. $c_{n}{ }^{*} c_{n}$ is obviously the well-known Poisson probability distribution function. Substitute (I6), (I8) into (I7), use the Hermite polynomial generating function

$$
\exp \left(2 \xi \eta-\eta^{2}\right)=\sum_{n=0}^{\infty} \frac{H_{n}(\xi) \eta^{n}}{n !}
$$

separate exponent into its real and imaginary parts, and (I7) reduces to

$$
\begin{gathered}
\varphi(p, t)=\left(\frac{\alpha}{\sqrt{ } \pi}\right)^{\frac{1}{2}} \exp \left\{-\frac{\alpha^{2}}{2}\left[p-\frac{(2 \lambda)^{\frac{1}{2}}}{\alpha} \cos (\omega t+\varphi)\right]^{2}\right\} \\
\times \exp \left\{i \left[\frac{\lambda}{2} \sin 2(\omega t+\varphi)\right.\right. \\
\left.\left.-\alpha p(2 \lambda)^{\frac{1}{2}} \sin (\omega t+\varphi)-\frac{\omega t}{2}\right]\right\} .
\end{gathered}
$$

This is in the form of a Gaussian distribution and our next task is to show that this is a minimum uncertainty wave function. That is, we must show that

where

$$
(\Delta p)^{2}(\Delta q)^{2}=\hbar^{2} / 4
$$

$$
\begin{aligned}
& (\Delta p)^{2}=\left\langle(p-\langle p\rangle)^{2}\right\rangle=\left\langle p^{2}\right\rangle-\langle p\rangle^{2}, \\
& (\Delta q)^{2}=\left\langle(q-\langle q\rangle)^{2}\right\rangle=\left\langle q^{2}\right\rangle-\langle q\rangle^{2} .
\end{aligned}
$$

Now it is easy to show that

$$
\begin{aligned}
\langle p\rangle & =\int_{-\infty}^{\infty}|\varphi(p, t)|^{2} p d p=\frac{(2 \lambda)^{\frac{1}{2}}}{\alpha} \cos (\omega t+\varphi), \\
\langle q\rangle & =\int_{-\infty}^{\infty} \varphi^{*} i \hbar \frac{\partial \varphi}{\partial p} d p=\hbar \alpha(2 \lambda)^{\frac{1}{2}} \sin (\omega t+\varphi), \\
\left\langle p^{2}\right\rangle & =\langle p\rangle^{2}+1 / 2 \alpha^{2}, \\
\left\langle q^{2}\right\rangle & =\langle q\rangle^{2}-\hbar^{2} \alpha^{4}\left(\left\langle p^{2}\right\rangle-\langle p\rangle^{2}\right)+\hbar^{2} \alpha^{2}
\end{aligned}
$$

from which (I11) follows directly showing (I10) to be a minimum uncertainty wave packet.

To demonstrate Ehrenfest's theorem, we see by Eqs. (I1), (I13), and (I14) that

$$
\begin{aligned}
& \langle\mathbf{E}(\mathbf{r}, t)\rangle=-(8 \pi \lambda \hbar \omega)^{\frac{1}{2}} \cos (\omega t+\varphi) \mathbf{u}(\mathbf{r}), \\
& \langle\mathbf{H}(\mathbf{r}, t)\rangle=\left(8 \pi \lambda \hbar c^{2} / \omega\right)^{\frac{1}{2}} \sin (\omega t+\varphi) \operatorname{curl} \mathbf{u}(\mathbf{r}),
\end{aligned}
$$

which shows that the expectation values of the fields for the "Poisson-state" yield the classical fields. 
Furthermore, if we denote the classical field amplitude by $E_{0}$ we see that

$$
\hbar \omega \lambda=E_{0}^{2} / 8 \pi,
$$

which is the classical field energy density which justifies the interpretation of $\lambda$ as the average number of photons in the field.

It is not surprising that the Poisson distribution which leads to the minimum uncertainty product leads to the classical result since this corresponds to localizing the photons as much as possible consistent with the uncertainty relations as well as specifying their momenta. This represents, therefore, the closest approach to the classical situation in which both position and momentum can be specified exactly and is a justification for assuming that the amplifier input has a Poisson distribution.

\section{APPENDIX II}

In order to simplify the evaluation of matrix elements, we begin by rewriting the Poisson distribution as an operator on the vacuum state. For one amplifier channel, the initial state may be written as

since

$$
|\psi(0)\rangle=\sum_{n=0}^{\infty}\left(\frac{e^{-\lambda} \lambda^{n}}{n !}\right)^{\frac{1}{2}} e^{-i n \varphi} \frac{a^{\dagger n}}{\sqrt{ } n !}|0\rangle,
$$

$$
|n\rangle=\left(a^{\dagger n} / \sqrt{ } n !\right)|0\rangle,
$$

where $|0\rangle$ is the vacuum state for this channel and we have used Eq. (12) $n$ times. Equation (II1) may be rewritten as

$$
\begin{aligned}
|\psi(0)\rangle & =e^{-\lambda / 2} \sum_{n=0}^{\infty} \frac{\left((\lambda)^{\frac{1}{2}} e^{-i \varphi} a^{\dagger}\right)^{n}}{n !}|0\rangle \\
& =e^{-\lambda / 2} \exp \left(w a^{\dagger}\right)|0\rangle
\end{aligned}
$$

where we have summed the series and

$$
w=(\lambda)^{\frac{1}{2}} e^{-i \varphi} \text {. }
$$

Note that $\lambda=\bar{n}$, the average number of photons in the channel.

We may generalize this result to two channels and write the initial wave function as

$$
|\psi(0)\rangle=e^{-\frac{1}{2}\left(\lambda_{1}+\lambda_{2}\right)} \exp \left(w_{1} a_{10}^{\dagger}\right)|0\rangle \exp \left(w_{2} a_{20}^{\dagger}\right)|0\rangle
$$

where

$$
w_{1,2}=\left(\lambda_{1,2}\right)^{\frac{1}{2}} e^{-i \varphi_{1,2}} .
$$

We now proceed to evaluate a typical matrix element, say $\left\langle\psi(0)\left|a_{10}{ }^{\dagger} a_{20}{ }^{\dagger}\right| \psi(0)\right\rangle$. Using Eq. (II5) this becomes

$$
\begin{aligned}
\left\langle\psi(0)\left|a_{10}^{\dagger} a_{20}{ }^{\dagger}\right| \psi(0)\right\rangle & =e^{-\left(\lambda_{1}+\lambda_{2}\right)}\left\langle 0\left|\exp \left(w_{1}^{*} a_{10}\right) a_{10}{ }^{\dagger} \exp \left(w_{1} a_{10}{ }^{\dagger}\right)\right| 0\right\rangle \\
& \times\left\langle 0\left|\exp \left(w_{2}{ }^{*} a_{20}\right) a_{20}{ }^{\dagger} \exp \left(w_{2} a_{20}{ }^{\dagger}\right)\right| 0\right\rangle .
\end{aligned}
$$

To evaluate we use the following theorem:

Theorem 1. If $f\left(a^{\dagger}\right)$ can be expanded in a Taylor series and $u$ is a parameter, then

$$
e^{u a} f\left(a^{\dagger}\right)|0\rangle=f\left(a^{\dagger}+u\right)|0\rangle .
$$

To prove this theorem, we note that since $\left[a, a^{\dagger}\right]=1$, $a$ can be replaced by

$$
a=\partial / \partial a^{\dagger},
$$

since $a$ and $a^{\dagger}$ are conjugate variables.

Now it is well known that a Taylor series expansion of $f(x)$ may be written as

$$
\begin{aligned}
f(x)=\exp \left[\left(x-x_{0}\right) \frac{\partial}{\partial x}\right] & f\left(x_{0}\right) \\
& =\sum_{l=0}^{\infty} \frac{\left(x-x_{0}\right)^{l}}{l !} \frac{\partial^{l}}{\partial x^{l}} f\left(x_{0}\right),
\end{aligned}
$$

and if $u=x-x_{0}$, we have

$$
f\left(u+x_{0}\right)=\exp \left(u \frac{\partial}{\partial x}\right) f\left(x_{0}\right)
$$

so that

$$
\exp \left(u \frac{\partial}{\partial a^{\dagger}}\right) f\left(a^{\dagger}\right)=f\left(a^{\dagger}+u\right),
$$

and the theorem is proved. Thus, the matrix element becomes

$$
\begin{aligned}
& \left\langle\psi(0)\left|a_{10}{ }^{\dagger} a_{20}{ }^{\dagger}\right| \psi(0)\right\rangle \\
& =e^{-\left(\lambda_{1}+\lambda_{2}\right)}\left\langle 0\left|\left(a_{10}{ }^{\dagger}+w_{1}^{*}\right) \exp \left[w_{1}\left(a_{10}{ }^{\dagger}+w_{1}^{*}\right)\right]\right| 0\right\rangle \\
& \quad \times\left\langle 0\left|\left(a_{20}{ }^{\dagger}+w_{2}^{*}\right) \exp \left[w_{2}\left(a_{20}{ }^{\dagger}+w_{2}^{*}\right)\right]\right| 0\right\rangle \\
& =w_{1}^{*} w_{2}^{*}=\left(\lambda_{1} \lambda_{2}\right)^{\frac{2}{2}} e^{-i\left(\varphi_{1}+\varphi_{2}\right)}
\end{aligned}
$$

since

$\left\langle 0\left|a_{10}^{\dagger} \exp \left(w_{1} a_{10}^{\dagger}\right)\right| 0\right\rangle$

$$
\begin{array}{r}
=\left\langle 0\left|a_{10} \dagger \sum_{l=0}^{\infty} \frac{\left(w_{1} a_{10^{\dagger}}\right)^{l}}{l !}\right| 0\right\rangle=0, \\
\left\langle 0\left|\exp \left(w_{1} a_{10}^{\dagger}\right)\right| 0\right\rangle=\left\langle 0\left|\sum_{l=0}^{\infty} \frac{\left(w_{1} a_{10^{\dagger}}\right)^{l}}{l !}\right| 0\right\rangle=1,
\end{array}
$$

and

$$
\left|w_{1}\right|^{2}=\lambda_{1},
$$

where $\lambda_{1}=\bar{n}_{10}$ and $\lambda_{2}=\bar{n}_{20}$, giving the desired matrix element. 